\title{
Medical Product Stability Date
}

National Cancer Institute

\section{Source}

National Cancer Institute. Medical Product Stability Date. NCI Thesaurus. Code C71365.

A date until which the medical product retains its properties within specified limits, and resists conditions that may affect its stated performance. After it is activated, the product is considered useable during the stability period. 